Research Article

\title{
Study of Convergence of Reduced Differential Transform Method for Different Classes of Differential Equations
}

\author{
Seyyedeh Roodabeh Moosavi Noori (i) and Nasir Taghizadeh \\ Department of Pure Mathematics, Faculty of Mathematical Sciences, University of Guilan, P.O.Box 1914, Rasht, Iran \\ Correspondence should be addressed to Seyyedeh Roodabeh Moosavi Noori; moosavinoori@gmail.com
}

Received 16 October 2020; Revised 29 March 2021; Accepted 19 April 2021; Published 30 April 2021

Academic Editor: Mostafa Eslami

Copyright ( $\odot 2021$ Seyyedeh Roodabeh Moosavi Noori and Nasir Taghizadeh. This is an open access article distributed under the Creative Commons Attribution License, which permits unrestricted use, distribution, and reproduction in any medium, provided the original work is properly cited.

In this work, we study the sufficient condition for convergence of the reduced differential transform method for nonlinear differential equations. The main power of this method is its ability and flexibility in solving linear and nonlinear problems properly and easily and obtain solutions both numerically and analytically. Simple approaches of reduced differential transform method and the convergence results for different classes of differential equations such as linear and nonlinear ordinary, partial, fractional, and system of differential equations are briefly discussed. Eight examples are checked to confirm convergence results as well as the strength and efficiency of the method.

\section{Introduction}

The reduced differential transform method (RDTM) is an analytical-numerical technique introduced for the first time by Keskin $[1,2]$ to study the analytical solutions of linear and nonlinear wave equations $[3,4]$. This suggested technique is highly efficient and powerful in obtaining the exact solutions as well as approximate solutions of mathematical modeling of many problems in technology, finance, engineering disciplines, natural sciences such as biology, physics, chemistry, and Earth science, gives the solution in the form of rapidly convergent successive approximations, and is capable of handling linear and nonlinear equations in a similar manner. In recent years, the reduced differential transform method has been widely adopted by many researchers such as in [5-9] and by the references therein. Also, it was shown by many authors [10-14] that the solution procedure of the RDTM is simpler and more straightforward than the homotopy perturbation method (HPM), differential transform method (DTM), variational iteration method (VIM), Adomian decomposition method (ADM), etc. On the contrary, the size of computational work has been reduced while still maintaining high precision from the numerical solution and rapid convergence has been guaranteed. The advantage of this method is its simplicity in using, and it solves the equations straightforward and directly without using Adomian's polynomial, perturbation, linearization or any other transformation, and restrictive conditions and gives the solution as convergent power series with simply determinable components. Therefore, the RDTM can overcome the foregoing limitations and restrictions of perturbation techniques and complicated computational so that it provides us with a possibility to analyse accurately nonlinear equations. The RDTM was successfully applied to ordinary differential equations [15], partial differential equations [16-18], fractional differential equations [19-23], Volterra integral equation [24-26], and integro-differential equations [27-29].

In the present work, in light of the abovementioned method, we will study linear and nonlinear problems. In addition, our main objective is to study the sufficient condition for convergence of the method for nonlinear equations. The main ideas explained in this paper are expected to be used for more linear and nonlinear models.

The rest of this study is presented as follows. In Section 2, we simply introduce the reduced differential transform method (RDTM). In Section 3, we prove the convergences of the considered method. In Section 4, RDTM approaches and 
convergence results are addressed. In Section 5, we apply this method to obtain the exact solutions for linear and nonlinear ordinary, partial, fractional, and system of differential equations. Finally, we offer some summaries and conclusions in Section 6.

\section{Summary of the Method}

We present some important definitions and mathematical preliminaries operations of the reduced differential transform method in which can help to more understand of the stated method in this section. Now, consider the function of two variables $w(x, t)$ and assume that it can be expressed as the product of two different variable functions, i.e., $w(x, t)=\phi(x) \psi(t)$. The function $w(x, t)$ can be displayed due to the properties of differential transform as follows:

$$
w(x, t)=\left(\sum_{i=0}^{\infty} \Phi(i) x^{i}\right)\left(\sum_{j=0}^{\infty} \Psi(j) t^{j}\right)=\sum_{k=0}^{\infty} \mathscr{W}_{k}(x) t^{k}
$$

where $\mathscr{W}_{k}(x)$ is the $t$-dimensional spectrum function of the original function $w(x, t)$.

Definition 1. The reduced differential transform function of $w(x, t)$ can be yield in the following form:

$$
\mathscr{W}_{k}(x)=\frac{1}{k !}\left[\frac{\partial^{k}}{\partial t^{k}} w(x, t)\right]_{t=t_{0}},
$$

where $w(x, t)$ is analytic and differentiated continuously function with regard to space $x$ and time $t$, in the domain of interest.

Definition 2. The reduced differential inverse transform of $\mathscr{W}_{k}(x)$ is determined as

$$
w(x, t)=\sum_{k=0}^{\infty} \mathscr{W}_{k}(x)\left(t-t_{0}\right)^{k} .
$$

Afterward, consolidating equations (2) and (3) yields

$$
w(x, t)=\sum_{k=0}^{\infty} \frac{1}{k !}\left[\frac{\partial^{k}}{\partial t^{k}} w(x, t)\right]_{t=t_{0}}\left(t-t_{0}\right)^{k} .
$$

By the help of the upper definitions, to illustrate the basic idea of the RDTM, consider the following operator form of nonlinear partial differential equations written as

$$
\mathscr{L} w(x, t)+\mathscr{R} w(x, t)+\mathscr{N} w(x, t)=h(x, t),
$$

with considering the following initial condition:

$$
w(x, 0)=g(x)
$$

where $\mathscr{L}=\partial / \partial t, \mathscr{R}$ and $\mathscr{N}$ indicate linear and nonlinear operators which have partial derivatives, and $h$ is a nonhomogeneous term.

After applying the RDTM definition on both sides of equation (5), we can write the following iteration formula:

$$
(k+1) \mathscr{W}_{k+1}(x)=\mathscr{H}_{k}(x)-\mathscr{R} \mathscr{W}_{k}(x)-\mathscr{N} \mathscr{W}_{k}(x),
$$

where $\mathscr{L} \mathscr{W}_{k}(x), \mathscr{R} \mathscr{W}_{k}(x), \mathscr{N} \mathscr{W}_{k}(x)$, and $\mathscr{H}_{k}(x)$ are the reduced differential transform functions of $\mathscr{L} w(x, t)$, $\mathscr{R} w(x, t), \mathcal{N} w(x, t)$, and $h(x, t)$, respectively.

Implementing the aforesaid method to the initial condition (6), we have

$$
\mathscr{W}_{0}(x)=g(x)
$$

To discover the remaining iteration, we plug equation (8) into (7) by simple reiterative calculation, and we tend to get the subsequent $\mathscr{W}_{k}(x)$ values. Afterwards, the inverse transformation of the set of values $\left\{\mathscr{W}_{k}(x)\right\}_{k=0}^{n}$ admits the $n$-terms approximation solution as follows:

$$
\widetilde{w}_{n}(x, t)=\sum_{k=0}^{n} \mathscr{W}_{k}(x)\left(t-t_{0}\right)^{k}
$$

Thus, the exact solution of the considered problem can be gained by

$$
w(x, t)=\lim _{n \longrightarrow \infty} \widetilde{w}_{n}(x, t)
$$

Table 1 contains the basic mathematical operations carried out by RDTM.

\section{Convergence of Method}

The principal aim of this section is to survey the sufficient conditions for convergence of the reduced differential transform method, according to the approach described by this method for solving the nonlinear equation (5) in Section 2. For this purpose, some theorems for convergence of the method and the error computation are addressed.

The fundamental point views of RDTM for the solutions of nonlinear models include ascertaining power series expansion with the initial time $t_{0}$ :

$$
w(x, t)=\sum_{k=0}^{\infty} a_{k}(x)\left(t-t_{0}\right)^{k}, \quad t \in l,
$$

where $l=\left(t_{0}, t_{0}+r\right), r>0$. The important results are proposed in the following theorems.

Theorem 1. If $\varphi_{k}(x, t)=a_{k}(x)\left(t-t_{0}\right)^{k}$, then the series solution $\sum_{k=0}^{\infty} \varphi_{k}(x, t)$, stated in equation (11), $\forall k \in \mathbf{N} \cup\{0\}$.

(i) It is convergent if $\exists 0<\lambda<1$ such that $\left\|\varphi_{k+1}\right\| \leq \lambda\left\|\varphi_{k}\right\|$

(ii) It is divergent if $\exists \lambda>1$ such that $\left\|\varphi_{k+1}\right\| \geq \lambda\left\|\varphi_{k}\right\|$

Theorem 1 is a specific case of Banach's fixed point theorem. Using this theorem and a brief description of its proof, we investigate the truncation error of the series solution equation (11) as follows.

Proof. Let $(C[l],\|\|$.$) be the Banach space of all continuous$ functions on $l$ with the norm $\left\|\varphi_{k}(x, t)\right\|=\left\|a_{k}(x)\left(t-t_{0}\right)^{k}\right\|$. Also, assume that $\left\|a_{0}(x)\right\|<N_{0}$, where $N_{0}$ is a positive number. Define the sequence of partial sums $\left\{\mathfrak{S}_{n}\right\}_{n=0}^{\infty}$ as

$$
\mathfrak{\Im}_{n}=\varphi_{0}+\varphi_{1}+\cdots+\varphi_{n}
$$


TABLE 1: The fundamental operations of RDTM.

\begin{tabular}{lr}
\hline Original form & \multicolumn{1}{c}{ Transformed form } \\
\hline$w(x, t)$ & $\mathscr{W}_{k}(x)=1 / k !\left[\partial^{k} / \partial t^{k} w(x, t)\right]_{t=0}$ \\
\hline$w(x, t)=\lambda u(x, t) \pm \gamma v(x, t)$ & $\mathscr{W}_{k}(x)=\lambda \mathscr{U}_{k}(x) \pm \gamma \mathscr{V}_{k}(x)(\lambda, \gamma$ are constants) \\
\hline$w(x, t)=x^{m} t^{n}$ & $\mathscr{W}_{k}(x)=x^{m} \delta(k-n), \delta(x)=\left\{\begin{array}{l}1, k=0 \\
0, k \neq 0\end{array}\right.$ \\
\hline$w(x, t)=x^{m} t^{n} u(x, t)$ & $\mathscr{W}_{k}(x)=x^{m} \mathscr{U}_{k-n}(x)$ \\
\hline$w(x, t)=u(x, t) v(x, t)$ & $\mathscr{W}_{k}(x)=\sum_{r=0}^{k} \mathscr{U}_{r}(x) \mathscr{V}_{k-r}(x)=\sum_{r=0}^{k} \mathscr{V}_{r}(x) \mathcal{U}_{k-r}(x)$ \\
\hline$w(x, t)=\partial^{r} / \partial t^{r} u(x, t)$ & $\mathscr{W}_{k}(x)=(k+1) \ldots(k+r) \mathscr{U}_{k+r}(x)=(k+r) ! / k ! \mathscr{U}_{k+r}(x)$ \\
\hline$w(x, t)=\partial / \partial x u(x, t)$ & $\mathscr{W}_{k}(x)=\partial / \partial x \mathscr{U}_{k}(x)$ \\
\hline$w(x, t)=e^{\lambda t}$ & $\mathscr{W}_{k}(x)=\lambda^{k} / k !$ \\
\hline$w(x, t)=\sin (\lambda t+\alpha x)$ & $\mathscr{W}_{k}(x)=\lambda^{k} / k ! \sin (k \pi / 2+\alpha x)$ \\
\hline$w(x, t)=\cos (\lambda t+\alpha x)$ & $\mathscr{W}_{k}(x)=\lambda^{k} / k ! \cos (k \pi / 2+\alpha x)$ \\
\hline
\end{tabular}

We want to show that $\left\{\widetilde{S}_{n}\right\}_{n=0}^{\infty}$ is a Cauchy sequence in this Banach space. To reach this goal, we take

$$
\left\|\mathfrak{S}_{n+1}-\mathfrak{S}_{n}\right\|=\left\|\varphi_{n+1}\right\| \leq \lambda\left\|\varphi_{n}\right\| \leq \cdots \leq \lambda^{n+1}\left\|\varphi_{0}\right\| \leq \lambda^{n+1} N_{0} .
$$

Therefore, for any $n, m \in \mathbb{N}, n \geq m$, making use of (13) and the triangle inequality successively, we have

$$
\begin{aligned}
\left\|\mathfrak{S}_{n}-\mathfrak{S}_{m}\right\| & =\left\|\left(\mathfrak{S}_{n}-\mathfrak{S}_{n-1}\right)+\left(\mathfrak{S}_{n-1}-\mathfrak{S}_{n-2}\right)+\cdots+\left(\mathfrak{S}_{m+1}-\mathfrak{S}_{m}\right)\right\| \\
& \leq\left\|\left(\mathfrak{S}_{n}-\mathfrak{S}_{n-1}\right)\right\|+\left\|\left(\mathfrak{S}_{n-1}-\mathfrak{S}_{n-2}\right)\right\|+\cdots+\left\|\left(\mathfrak{S}_{m+1}-\mathfrak{S}_{m}\right)\right\| \\
& \leq \frac{1-\lambda^{n-m}}{1-\lambda} \lambda^{m+1}\left\|\varphi_{0}\right\|,
\end{aligned}
$$

and because $0<\lambda<1$, we obtain

$$
\lim _{n, m \rightarrow \infty}\left\|\mathfrak{S}_{n}-\mathfrak{S}_{m}\right\|=0 .
$$

Hence, $\left\{\widetilde{\mathfrak{S}}_{n}\right\}_{n=0}^{\infty}$ is a Cauchy sequence in the Banach space $(C[l],\|\|$.$) . Thus, the series solution \sum_{k=0}^{\infty} \varphi_{k}(x, t)$, defined in equation (11), is convergent and it completes the proof.

Remark 1. According to the assumptions in (ii) and by using the ratio test, we have

$$
\left\|\frac{\varphi_{k+1}}{\varphi_{k}}\right\| \geq \lambda>1
$$

As a result, the series is divergent.

Remark 2. If the series $\sum_{k=0}^{\infty} a_{k}(x)\left(t-t_{0}\right)^{k}$ of the nonlinear equation (5) converges, then it is an exact solution.

Theorem 2. Suppose that the series solution $\sum_{k=0}^{\infty} \varphi_{k}(x, t)$, where $\varphi_{k}(x, t)=a_{k}(x)\left(t-t_{0}\right)^{k}$, converges to the solution $w(x, t)$. If the truncated series $\sum_{k=0}^{m} \varphi_{k}(x, t)$ is used as an approximation to the solution $u(x, t)$, then the maximum absolute truncated error is estimated as

$$
\left\|w(x, t)-\sum_{k=0}^{m} \varphi_{k}(x, t)\right\| \leq \frac{1}{1-\lambda} \lambda^{m+1}\left\|\varphi_{0}\right\| .
$$

Proof. According to Theorem 1, we have the inequality equation (14) as follows:

$$
\left\|\mathfrak{S}_{n}-\mathfrak{S}_{m}\right\| \leq \frac{1-\lambda^{n-m}}{1-\lambda} \lambda^{m+1}\left\|\varphi_{0}\right\|
$$

for $n \geq m$. Also, since $0<\lambda<1$, in the numerator, we have $1-\lambda^{n-m}<1$; therefore, the inequality equation (18) can be reduced to

$$
\left\|\mathfrak{S}_{n}-\mathfrak{S}_{m}\right\| \leq \frac{1}{1-\lambda} \lambda^{m+1}\left\|\varphi_{0}\right\|
$$

It is clear when $n \longrightarrow \infty, \mathfrak{S}_{n} \longrightarrow w(x, t)$. Thus, inequality equation (17) is obtained and the theorem is proved.

In summary, Theorems 1 and 2 state that the reduced differential transform solution of nonlinear equation (5), obtained using the iteration formula (7) and (8), converges to an exact solution under the condition that $\exists 0<\lambda<1$ such that $\left\|\varphi_{k+1}\right\| \leq \lambda\left\|\varphi_{k}\right\|, \forall k \in \mathbb{N} \cup\{0\}$. In other words, if we define, for every $i \in \mathbb{N} \cup\{0\}$, the parameters,

$$
\gamma_{i}= \begin{cases}\frac{\left\|\varphi_{i+1}\right\|}{\left\|\varphi_{i}\right\|}, & \left\|\varphi_{i}\right\| \neq 0, \\ 0, & \left\|\varphi_{i}\right\|=0,\end{cases}
$$


then the series solution $\sum_{k=0}^{\infty} \varphi_{k}(x, t)$ of equation (5) converges to an exact solution $w(x, t)$, when $0 \leq \gamma_{i}<1, \forall i \in \mathbb{N} \cup\{0\}$. In addition, the maximum absolute truncation error, as discussed in Theorem 2, is estimated to be

$$
\left\|w(x, t)-\sum_{k=0}^{m} \varphi_{k}(x, t)\right\| \leq \frac{1}{\gamma-1} \gamma^{j+1}\left\|\varphi_{0}\right\|,
$$

where $\gamma=\max \left\{\gamma_{i}, i=0,1, \ldots, j\right\}$.

Remark 3. The first finite terms have no effect the convergence of the series solution. In other words, if the first finite $\gamma_{i} s, i=0,1, \ldots, l$, are not less than one and $\gamma_{i} \leq 1$ for $i>l$, then the series solution $\sum_{k=0}^{\infty} \varphi_{k}(x, t)$ of equation (5) converges to an exact solution. Because, according to Theorem 1, we have

$$
\left\|\mathfrak{S}_{n}-\mathfrak{S}_{j}\right\| \leq \frac{1-\lambda^{n-j}}{1-\lambda} \lambda^{j-l}\left\|\varphi_{l+1}\right\|
$$

and since $0<\lambda<1$, for $n \geq j$ and fixed $l$, we get $\lim _{n, j \rightarrow \infty}$ $\left\|\mathfrak{S}_{n}-\mathfrak{S}_{j}\right\|=0$. In this case, the convergence of the RDTM approach depends on $\gamma_{i}$, for $i>l$.

\section{RDTM Approaches and Convergence Results}

The principal aim of this section is to summarize RDTM inclusive convergence results of the method for solving different classes of differential equations.

4.1. Ordinary Differential Equations (ODE). Let us write the nonlinear ordinary differential equation as the following:

$$
\frac{\mathrm{d}^{r}}{\mathrm{~d} t^{r}} w(t)+\mathscr{R} w(t)+\mathscr{N} w(t)=h(t), \quad t>0,
$$

where $r \in \mathbb{N}, \mathscr{R}$ is a linear operator, $\mathcal{N}$ is a nonlinear operator, and $h$ is a nonhomogeneous term, with the initial conditions $w^{(k)}(0)=c_{k}, k=0,1, \ldots, r-1$. According to the operations of differential transformation, the series solution $w(t)=\sum_{k=0}^{\infty} \varphi_{k}(t)$ is obtained using the following iteration formula:

$$
\frac{(k+r) !}{k !} \mathscr{W}(k+r)+\mathscr{R} \mathscr{W}(k)+\mathscr{N} \mathscr{W}(k)=\mathscr{H}(k),
$$

which converges to a solution of equation (23) if $\forall k \in \mathbb{N} \cup\{0\}$, $\exists 0<\lambda<1$ such that $\left\|\varphi_{k+1}\right\| \leq \lambda\left\|\varphi_{k}\right\|$.

4.2. Partial Differential Equations (PDE). Let us write the nonlinear partial differential equation as the following:

$$
\frac{\partial^{r}}{\partial t^{r}} w(x, t)+\mathscr{R} w(x, t)+\mathscr{N} w(x, t)=h(x, t), \quad t>0,
$$

where $r \in \mathbb{N}, \mathscr{R}$ is a linear operator, $\mathcal{N}$ is a nonlinear operator, and $h$ is a nonhomogeneous term, with the initial conditions $w^{(k)}(x, 0)=g_{k}(x), k=0,1, \ldots, r-1$. According to the operations of differential transformation, the series solution $w(x, t)=\sum_{k=0}^{\infty} \varphi_{k}(x, t)$ is obtained using the following iteration formula:

$$
\frac{(k+r) !}{k !} \mathscr{W}_{k+r}(x)+\mathscr{R} \mathscr{W}_{k}(x)+\mathscr{N} \mathscr{W}_{k}(x)=\mathscr{H}_{k}(x)
$$

which converges to a solution of equation (25) if $\forall k \in \mathbb{N} \cup\{0\}, \exists 0<\lambda<1$ such that $\left\|\varphi_{k+1}\right\| \leq \lambda\left\|\varphi_{k}\right\|$.

4.3. Fractional Partial Differential Equations (FPDE). Let us write the nonlinear fractional partial differential equation as follows:

$$
\begin{gathered}
\frac{\partial^{\alpha r}}{\partial t^{\alpha r}} w(x, t)+\mathscr{R} w(x, t)+\mathcal{N} w(x, t) \\
\quad=h(x, t), \quad t>0, m-1<\alpha \leq m,
\end{gathered}
$$

where $m \in \mathbb{N}, \partial^{\alpha r} / \partial t^{\alpha r}$ is the Caputo fractional derivative of order $\alpha r, \mathscr{R}$ is a linear operator, $\mathcal{N}$ is a nonlinear operator, and $h$ is a nonhomogeneous term, with the initial conditions $w^{(k)}(x, 0)=g_{k}(x), k=0,1, \ldots, m-1$.

Definition 3. The Caputo fractional derivative operator is defined as

$$
\mathscr{D}_{a}^{\alpha} w(t)=\frac{1}{\Gamma(m-\alpha)} \int_{a}^{t}(t-\xi)^{m-\alpha-1} w^{(m)}(\xi) \mathrm{d} \xi,
$$

where $\alpha>0$ and $a$ are the order of the derivative and the initial value of function $w$, respectively.

Properties of Caputo fractional derivative operator can be found in [30-32].

Also, to determine the result, we introduce the subsequent Riemann-Liouville fractional integral operator.

Definition 4. The Riemann-Liouville fractional integral operator is defined as

$$
\mathcal{F}_{a}^{\alpha} w(t)=\frac{1}{\Gamma(\alpha)} \int_{a}^{t}(t-\xi)^{\alpha-1} w(\xi) \mathrm{d} \xi, \quad t>0, \alpha>0 .
$$

Properties of Riemann-Liouville fractional integral operator can be found in $[33,34]$.

Definition 5. Let function $w(x, t)$ be analytic and differentiated continuously with respect to space $x$ and time $t$ in the domain of interest, and the fractional reduced differential transform function (FRDTM) is

$$
\mathscr{W}_{k}^{\alpha}(x)=\frac{1}{\Gamma(\alpha k+1)}\left[\frac{\partial^{\alpha k}}{\partial t^{\alpha k}} w(x, t)\right]_{t=t_{0}},
$$

where $0<\alpha \leq 1$, the $t$-dimensional spectrum function $\mathscr{W}_{k}^{\alpha}(x)$ is the transformed function, and $\Gamma$ is gamma function is defined as

$$
\Gamma(p)=\int_{0}^{\infty} x^{p-1} e^{-x} \mathrm{~d} x
$$


Definition 6. The fractional reduced differential inverse transform of $W_{k}^{\alpha}(x)$ is determined as follows:

$$
w(x, t)=\sum_{k=0}^{\infty} \mathscr{W}_{k}^{\alpha}(x)\left(t-t_{0}\right)^{\alpha k}
$$

Afterward, combining equations (30) and (32), we write

$$
w(x, t)=\sum_{k=0}^{\infty} \frac{1}{\Gamma(\alpha k+1)}\left[\frac{\partial^{\alpha k}}{\partial t^{\alpha k}} w(x, t)\right]_{t=t_{0}}\left(t-t_{0}\right)^{\alpha k},
$$

which in practical application can be approximated by a finite series:

$$
\widetilde{w}_{n}(x, t)=\sum_{k=0}^{n} \mathscr{W}_{k}^{\alpha}(x)\left(t-t_{0}\right)^{\alpha k}
$$
by

Thus, the exact solution to the problem can be obtained

$$
w(x, t)=\lim _{n \longrightarrow \infty} \widetilde{w}_{n}(x, t) .
$$

In case of $\alpha=1$, FRDTM is reduced to classical RDTM. The basic properties of mathematical operations performed by FRDTM can be found in [33]. According to RDTM and Caputo differential operator and then following the same analysis presented in the previous section, the series solution $w(x, t)=\sum_{k=0}^{\infty} \varphi_{k}(x, t)$ is obtained using the following iteration formula:

$\frac{\Gamma(\alpha(k+r)+1)}{\Gamma(\alpha k+1)} \mathscr{W}_{k+r}^{\alpha}(x)+\mathscr{R} \mathscr{W}_{k}(x)+\mathscr{N} \mathscr{W}_{k}(x)=\mathscr{H}_{k}(x)$,

which converges to a solution of equation (27) if $\forall k \in \mathbb{N} \cup\{0\}$ and $\exists 0<\lambda<1$ such that $\left\|\varphi_{k+1}\right\| \leq \lambda\left\|\varphi_{k}\right\|$.

4.4. Systems of Fractional Partial Differential Equations. Let us write the following system of nonlinear fractional partial differential equations:

$$
\left\{\begin{array}{l}
\frac{\partial^{\alpha}}{\partial t^{\alpha}} w_{1}(x, t)+\mathscr{R}_{1}\left(w_{1}, w_{2}, \ldots, w_{n}\right)+\mathcal{N}_{1}\left(w_{1}, w_{2}, \ldots, w_{n}\right)=h_{1}(x, t) \\
\frac{\partial^{\alpha}}{\partial t^{\alpha}} w_{2}(x, t)+\mathscr{R}_{2}\left(w_{1}, w_{2}, \ldots, w_{n}\right)+\mathcal{N}_{2}\left(w_{1}, w_{2}, \ldots, w_{n}\right)=h_{2}(x, t), \\
\vdots \\
\frac{\partial^{\alpha}}{\partial t^{\alpha}} w_{n}(x, t)+\mathscr{R}_{n}\left(w_{1}, w_{2}, \ldots, w_{n}\right)+\mathcal{N}_{n}\left(w_{1}, w_{2}, \ldots, w_{n}\right)=h_{n}(x, t)
\end{array}\right.
$$

where $m-1<\alpha \leq m, m, n \in \mathbb{N}, \mathscr{R}_{1}, \mathscr{R}_{2}, \ldots, \mathscr{R}_{n}$ are linear operators, $\mathcal{N}_{1}, \mathcal{N}_{2}, \ldots, \mathcal{N}_{n}$ are nonlinear operators, and $h_{1}, h_{2}, \ldots, h_{n}$ are the nonhomogeneous terms, with the initial conditions

$$
\left\{\begin{array}{c}
w_{1}^{(k)}(x, 0)=g_{1, k}(x), \\
w_{2}^{(k)}(x, 0)=g_{2, k}(x), \\
\vdots \\
w_{n}^{(k)}(x, 0)=g_{n, k}(x),
\end{array}\right.
$$

for $k=0,1, \ldots, m-1$. Then, for $i=1,2, \ldots, n$, the series solution $w_{i}(x, t)=\sum_{k=0}^{\infty} \varphi_{i, k}(x, t)$ is obtained using the iteration formula:

$$
\frac{\Gamma(\alpha(k+r)+1)}{\Gamma(\alpha k+1)} \mathscr{W}_{i, k+1}^{\alpha}(x)+\mathscr{R} \mathscr{W}_{i, k}(x)+\mathscr{N} \mathscr{W}_{i, k}(x)=\mathscr{H}_{i, k}(x),
$$

which converges to a solution of equation (37) if $\forall k \in \mathbb{N} \cup\{0\}$ and $\exists 0<\lambda_{i}<1$ such that $\left\|\varphi_{i, k+1}\right\| \leq \lambda_{i}\left\|\varphi_{i, k}\right\|$. ?

\section{Applications}

The principal aim of this section is to apply the reduced differential transform method on the following examples to illustrate the accuracy of the presented method.

Example 1. We first consider the following linear ordinary differential equation,

$$
w^{\prime \prime}(t)+w(t)=0, \quad 0<t \leq 1,
$$

subject to the initial conditions:

$$
w(0)=0, w^{\prime}(0)=1 .
$$

According to the operations of differential transformation given in Table 1 for equation (40), we obtain the fol, lowing recurrent relation:

$$
(k+1)(k+2) \mathscr{W}(k+2)+\mathscr{W}(k)=0,
$$

and from initial conditions (42), we write

$$
\mathscr{W}(0)=0, \mathscr{W}(1)=1 .
$$


Substituting the above equations in equation (43), we drive the following results:

$$
\mathscr{W}(2)=0, \mathscr{W}(3)=-\frac{1}{3 !}, \mathscr{W}(4)=0, \mathscr{W}(5)=\frac{1}{5 !}, \cdots
$$

Hence, the solution in the series form is as follows:

$$
\begin{aligned}
\widetilde{w}_{n}(t) & =\sum_{k=0}^{\infty} \mathscr{W}(k) t^{k}=\mathscr{W}(0)+\mathscr{W}(1) t+\mathscr{W}(2) t^{2}+\cdots \\
& =t-\frac{t^{3}}{3 !}+\frac{t^{5}}{5 !}-\cdots,
\end{aligned}
$$

which converges efficiently to the exact solution $w(t)=\sin t$.

Example 2. As the second example, we consider the following nonlinear ordinary differential equation:

$$
w^{\prime}(t)=w^{2}(t)+1, \quad 0<t \leq 1,
$$

which subjects to the initial condition:

$$
w(0)=0 \text {. }
$$

According to the operations of differential transformation given in Table 1 for equation (46), we obtain the following recurrent relation:

$$
(k+1) \mathscr{W}(k+1)=\sum_{r=0}^{k} \mathscr{W}(r) \mathscr{W}(k-r)+\delta(k),
$$

and from initial condition (47), we write

$$
\mathscr{W}(0)=0 \text {. }
$$

Substituting the above equation in equation (48), we drive the following results:

$$
\begin{aligned}
& \mathscr{W}(1)=1, \mathscr{W}(2)=0, \mathscr{W}(3)=\frac{1}{3}, \mathscr{W}(4)=0, \\
& \mathscr{W}(5)=\frac{2}{15}, \ldots
\end{aligned}
$$

Hence, the solution in series form is as follows:

$$
\begin{aligned}
\widetilde{w}_{n}(t) & =\sum_{k=0}^{\infty} \mathscr{W}(k) t^{k}=\mathscr{W}(0)+\mathscr{W}(1) t+\mathscr{W}(2) t^{2}+\cdots \\
& =t+\frac{1}{3} t^{3}+\frac{2}{15} t^{5}+\cdots,
\end{aligned}
$$

which converges efficiently to the exact solution $w(t)=\tan t$.

Example 3. As the third example, we consider the following Burger's equation [35]:

$$
\frac{\partial w}{\partial t}+w \frac{\partial w}{\partial x}=\frac{\partial^{2} w}{\partial x^{2}}, \quad 0 \leq t<\frac{1}{2}
$$

which subjects to the initial condition:

$$
w(x, 0)=2 x .
$$

According to the operations of differential transformation given in Table 1 for equation (52), we obtain the following recurrent relation:

$$
(k+1) \mathscr{W}_{k+1}(x)+\sum_{r=0}^{k} \mathscr{W}_{r}(x) \frac{\partial}{\partial x} \mathscr{W}_{k-r}(x)=\frac{\partial^{2}}{\partial x^{2}} \mathscr{W}_{k}(x),
$$

and from initial condition (53), we write

$$
\mathscr{W}_{0}(x)=2 x .
$$

Substituting the above equation in equation (54), we drive the following results:

$$
\begin{aligned}
& \mathscr{W}_{1}(x)=-4 x, \mathscr{W}_{2}(x)=8 x, \mathscr{W}_{3}(x)=-16 x, \\
& \mathscr{W}_{4}(x)=32 x, \ldots
\end{aligned}
$$

Hence, the solution in the series form is as follows:

$$
\begin{aligned}
\widetilde{w}_{n}(x, t) & =\sum_{k=0}^{\infty} \mathscr{W}_{k}(x) t^{k}=\mathscr{W}_{0}(x)+\mathscr{W}_{1}(x) t+\mathscr{W}_{2}(x) t^{2}+\cdots \\
& =2 x\left(1-2 t+(2 t)^{2}-\cdots\right),
\end{aligned}
$$

which converges efficiently to the exact solution $w(x, t)=2 x / 1+2 t$.

Example 4. As the fourth example, we consider the following nonlinear Klein-Gordon equation [36]:

$$
\frac{\partial^{2} w}{\partial t^{2}}-\frac{\partial^{2} w}{\partial x^{2}}+w^{2}=-x \cos t+x^{2} \cos ^{2} t
$$

which subjects to the initial conditions:

$$
w(x, 0)=x, \frac{\partial}{\partial t} w(x, 0)=0 .
$$

According to the operations of differential transformation given in Table 1 for equation (58), we obtain the following recurrent relation:

$$
\begin{aligned}
& (k+1)(k+2) \mathscr{W}_{k+2}(x)-\frac{\partial^{2}}{\partial x^{2}} \mathscr{W}_{k}(x)+\sum_{r=0}^{k} \mathscr{W}_{r}(x) \mathscr{W}_{k-r}(x) \\
& =-x \frac{\cos k \pi / 2}{k !}+\frac{1}{2} x^{2} \delta(k)+2^{k} \frac{\cos k \pi / 2}{k !},
\end{aligned}
$$

and from initial conditions (59), we write

$$
\mathscr{W}_{0}(x)=x, \mathscr{W}_{1}(x)=0 .
$$

Substituting the above equation in equation (60), we drive the following results:

$$
\mathscr{W}_{2}(x)=-\frac{x}{2 !}, \mathscr{W}_{3}(x)=0, \mathscr{W}_{4}(x)=\frac{x}{4 !}, \mathscr{W}_{5}(x)=0, \ldots
$$

Hence, the solution in the series form is as follows: 


$$
\begin{aligned}
\widetilde{w}_{n}(x, t) & =\sum_{k=0}^{\infty} \mathscr{W}_{k}(x) t^{k}=\mathscr{W}_{0}(x)+\mathscr{W}_{1}(x) t+\mathscr{W}_{2}(x) t^{2}+\cdots \\
& =x\left(1-\frac{t^{2}}{2 !}+\frac{t^{4}}{4 !}-\cdots\right)
\end{aligned}
$$

which converges efficiently to the exact solution $w(x, t)=x \cos t$.

Example 5. As the fifth example, we consider the following homogeneous nonlinear time-fractional gas dynamics equation as $[37,38]$

$$
\frac{\partial^{\alpha} w}{\partial t^{\alpha}}+w \frac{\partial w}{\partial x}-w+w^{2}=0
$$

which subjects to the initial condition:

$$
w(x, 0)=e^{-x}
$$

$$
\begin{aligned}
& \widetilde{w}_{n}(x, t)=\sum_{k=0}^{\infty} \mathscr{W}_{k}^{\alpha}(x) t^{\alpha k} \\
& \left\{\begin{array}{l}
\frac{\partial w}{\partial t}-\frac{\partial v}{\partial x}-w+v=-2 \\
\frac{\partial v}{\partial t}+\frac{\partial w}{\partial x}-w+v=-2
\end{array}\right.
\end{aligned}
$$$$
=\mathscr{W}_{0}^{\alpha}(x)+\mathscr{W}_{1}^{\alpha}(x) t^{\alpha}+\mathscr{W}_{2}^{\alpha}(x) t^{2 \alpha}+\cdots=e^{-x}\left(1+\frac{1}{\Gamma(\alpha+1)} t^{\alpha}+\frac{1}{\Gamma(2 \alpha+1)} t^{2 \alpha}+\cdots\right),
$$

which for $\alpha=1$ converges efficiently to the exact solution $w(x, t)=e^{-x+t}$.

Example 6. As the sixth example, we consider the following system of inhomogeneous linear PDEs [33]:

which subjects to the initial condition:

$$
\left\{\begin{array}{l}
w(x, 0)=1+e^{x} \\
v(x, 0)=-1+e^{x}
\end{array}\right.
$$

According to the operations of differential transformation given in Table 1 for equation (70), we obtain the following recurrent relation:

$$
\begin{aligned}
& (k+1) \mathscr{W}_{k+1}(x)-\frac{\partial}{\partial x} \mathscr{V}_{k}(x)-\mathscr{W}_{k}(x)+\mathscr{V}_{k}(x)=-2 \delta(k), \\
& (k+1) \mathscr{V}_{k+1}(x)+\frac{\partial}{\partial x} \mathscr{W}_{k}(x)-\mathscr{W}_{k}(x)+\mathscr{V}_{k}(x)=-2 \delta(k),
\end{aligned}
$$

and from initial conditions (71), we write

$$
\begin{aligned}
& \mathscr{W}_{0}(x)=1+e^{x}, \\
& \mathscr{V}_{0}(x)=-1+e^{x}
\end{aligned}
$$
drive the following results:

$$
\begin{aligned}
& \mathscr{W}_{1}(x)=e^{x}, \\
& \mathscr{W}_{2}(x)=\frac{1}{2 !} e^{x}, \\
& \mathscr{W}_{3}(x)=\frac{1}{3 !} e^{x}, \\
& \mathscr{W}_{4}(x)=\frac{1}{4 !} e^{x}, \ldots, \\
& \mathscr{V}_{1}(x)=-e^{x}, \\
& \mathscr{V}_{2}(x)=\frac{1}{2 !} e^{x}, \\
& \mathscr{V}_{3}(x)=-\frac{1}{3 !} e^{x}, \\
& \mathscr{V}_{4}(x)=\frac{1}{4 !} e^{x}, \ldots
\end{aligned}
$$

According to the operations of differential transformation given in Table 1 for equation (64), we obtain the following recurrent relation:

$$
\begin{array}{r}
\frac{\Gamma(\alpha(k+1)+1)}{\Gamma(\alpha k+1)} \mathscr{W}_{k+1}^{\alpha}(x)+\sum_{r=0}^{k} \mathscr{W}_{r}(x) \frac{\partial}{\partial x} \mathscr{W}_{k-r}(x) \\
-\mathscr{W}_{k}+\sum_{r=0}^{k} \mathscr{W}_{r}(x) \mathscr{W}_{k-r}(x)=0,
\end{array}
$$

and from initial conditions (65), we write

$$
\mathscr{W}_{0}^{\alpha}(x)=e^{-x} \text {. }
$$

Substituting the above equation in equation (66), we drive the following results:

$$
\begin{aligned}
& \mathscr{W}_{1}^{\alpha}(x)=\frac{e^{-x}}{\Gamma(\alpha+1)}, \mathscr{W}_{2}^{\alpha}(x)=\frac{e^{-x}}{\Gamma(2 \alpha+1)}, \\
& \mathscr{W}_{3}^{\alpha}(x)=\frac{e^{-x}}{\Gamma(3 \alpha+1)}, \ldots
\end{aligned}
$$

Hence, the solution in series form is as follows:

Substituting the above equations in equation (72), we

Hence, the solutions in the series form are as follows: 


$$
\begin{aligned}
& \widetilde{w}_{n}(x, t)=\sum_{k=0}^{\infty} \mathscr{W}_{k}(x) t^{k}=\mathscr{W}_{0}(x)+\mathscr{W}_{1}(x) t+\mathscr{W}_{2}(x) t^{2}+\cdots=1+e^{x}\left(1+t+\frac{t^{2}}{2 !}+\cdots\right), \\
& \widetilde{v}_{n}(x, t)=\sum_{k=0}^{\infty} \mathscr{V}_{k}(x) t^{k}=\mathscr{V}_{0}(x)+\mathscr{V}_{1}(x) t+\mathscr{V}_{2}(x) t^{2}+\cdots=-1+e^{x}\left(1-t+\frac{t^{2}}{2 !}-\cdots\right),
\end{aligned}
$$

which converges efficiently to the exact solutions $w(x, t)=$ $1+e^{x+t}$ and $v(x, t)=-1+e^{x-t}$.

Example 7. As the seventh example, we consider the following system of nonlinear PDEs:

$$
\left\{\begin{array}{l}
\frac{\partial w}{\partial t}+\frac{\partial w}{\partial x} v-w \frac{\partial v}{\partial x}-w=0 \\
\frac{\partial v}{\partial t}-w \frac{\partial v}{\partial x}+\frac{\partial w}{\partial x} v+v=0
\end{array}\right.
$$

which subjects to the initial conditions:

$$
\left\{\begin{array}{l}
w(x, 0)=e^{x} \\
v(x, 0)=e^{x} .
\end{array}\right.
$$

According to the operations of differential transformation given in Table 1 for equation (76), we obtain the following recurrent relation:

$$
\begin{aligned}
& (k+1) \mathscr{W}_{k+1}(x)+\sum_{r=0}^{k} \frac{\partial}{\partial x} \mathscr{W}_{r}(x) \mathscr{V}_{k-r}-\sum_{r=0}^{k} \mathscr{W}_{r} \frac{\partial}{\partial x} \mathscr{V}_{k-r}(x)-\mathscr{W}_{k}(x)=0 \\
& (k+1) \mathscr{V}_{k+1}(x)-\sum_{r=0}^{k} \mathscr{W}_{r} \frac{\partial}{\partial x} \mathscr{V}_{k-r}(x)+\sum_{r=0}^{k} \frac{\partial}{\partial x} \mathscr{W}_{r}(x) \mathscr{V}_{k-r}+\mathscr{V}_{k}(x)=0
\end{aligned}
$$

and from initial conditions (77), we write

$$
\begin{aligned}
& \mathscr{W}_{0}(x)=e^{x}, \\
& \mathscr{V}_{0}(x)=e^{x}
\end{aligned}
$$

Substituting the above equations in equation (78), we drive the following results:

$$
\begin{aligned}
& \mathscr{W}_{1}(x)=e^{x}, \\
& \mathscr{W}_{2}(x)=\frac{1}{2 !} e^{x}, \\
& \mathscr{W}_{3}(x)=\frac{1}{3 !} e^{x},
\end{aligned}
$$

$$
\begin{gathered}
\widetilde{w}_{n}(x, t)=\sum_{k=0}^{\infty} \mathscr{W}_{k}(x) t^{k}=\mathscr{W}_{0}(x)+\mathscr{W}_{1}(x) t+\mathscr{W}_{2}(x) t^{2}+\cdots=e^{x}\left(1+t+\frac{t^{2}}{2 !}+\cdots\right), \\
\widetilde{v}_{n}(x, t)=\sum_{k=0}^{\infty} \mathscr{V}_{k}(x) t^{k}=\mathscr{V}_{0}(x)+\mathscr{V}_{1}(x) t+\mathscr{V}_{2}(x) t^{2}+\cdots=e^{x}\left(1-t+\frac{t^{2}}{2 !}-\cdots\right),
\end{gathered}
$$

which converges efficiently to the exact solutions $w(x, t)=$ $e^{x+t}$ and $v(x, t)=e^{x-t}$.
Example 8. Lastly, we consider the following system of homogeneous linear FPDEs [39, 40]: 


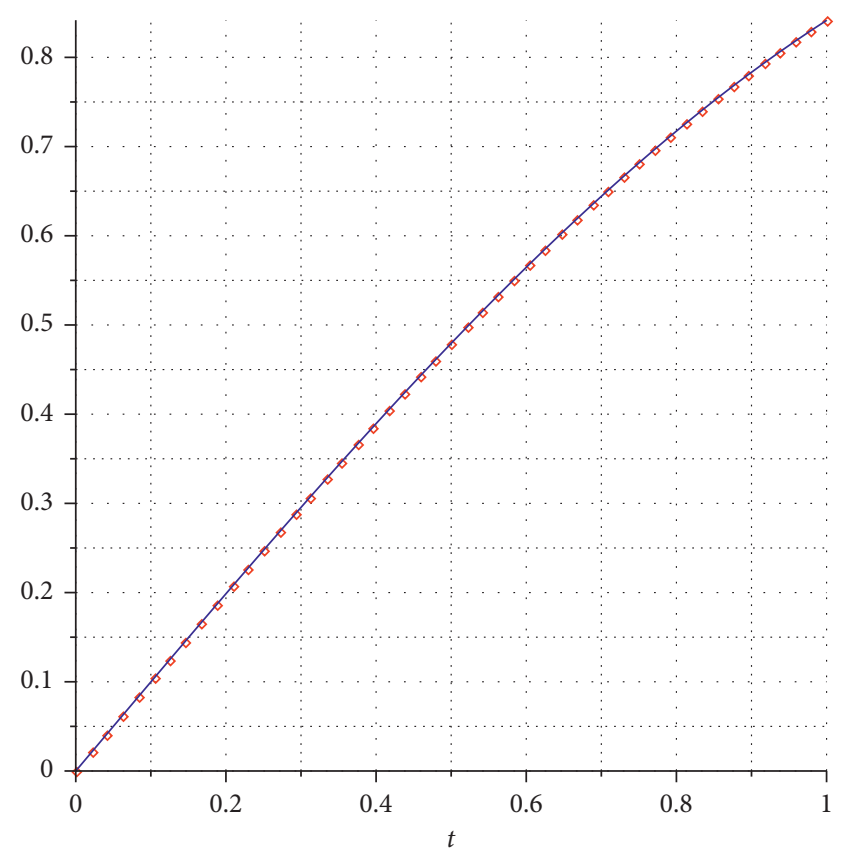

Figure 1: Comparison of the exact solution (blue) and the approximate solutions (red) of Example 1.

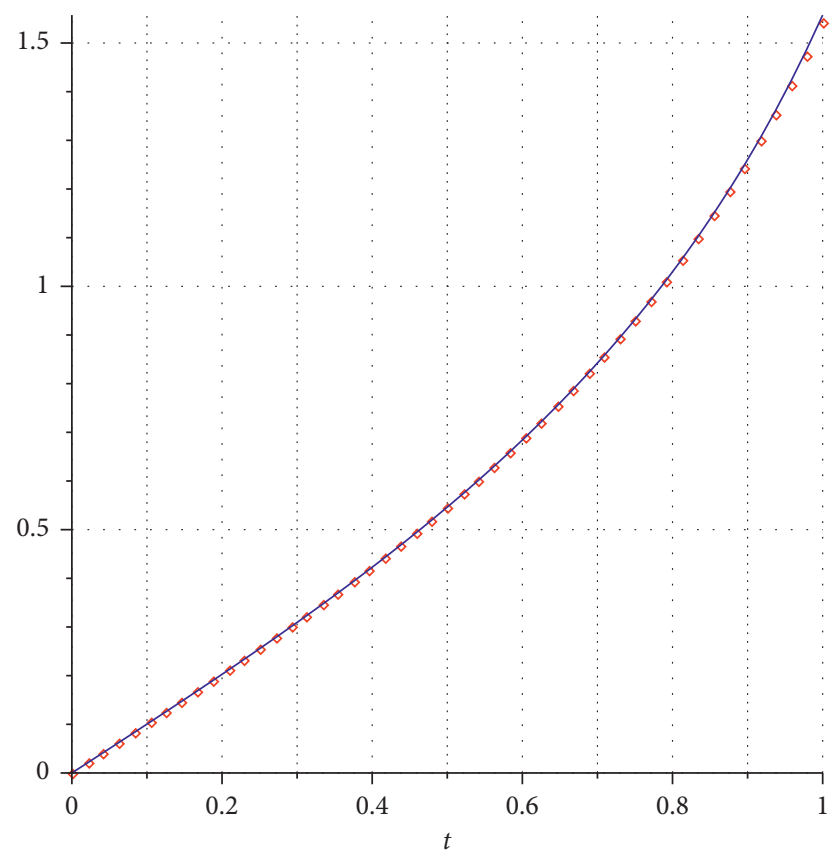

Figure 2: Comparison of the exact solution (blue) and the approximate solutions (red) of Example 2.

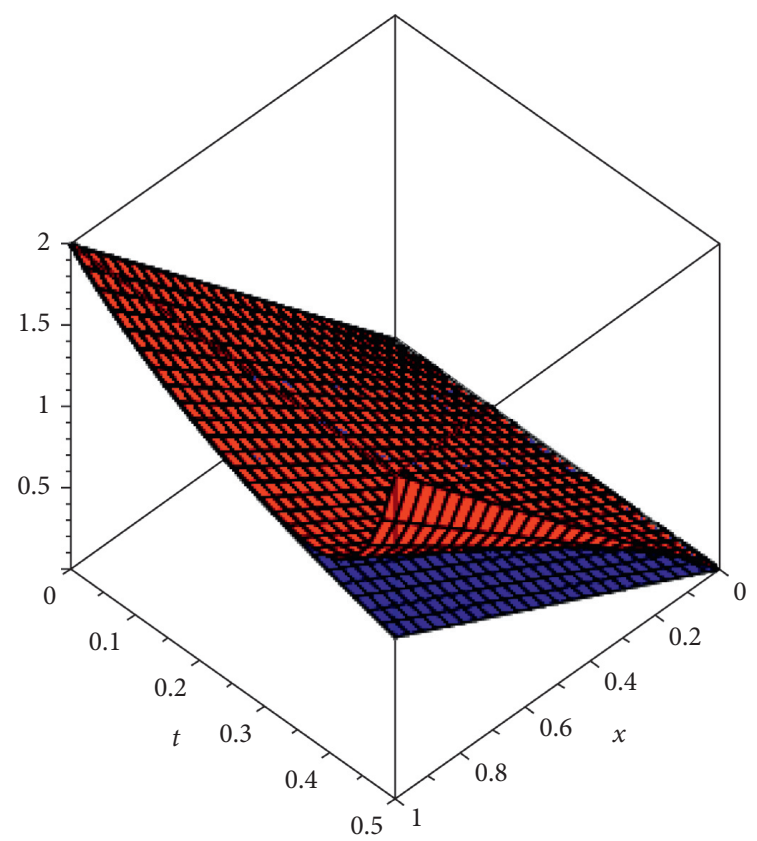

Figure 3: Comparison of the exact solution (blue) and the approximate solutions (red) of Example 3.

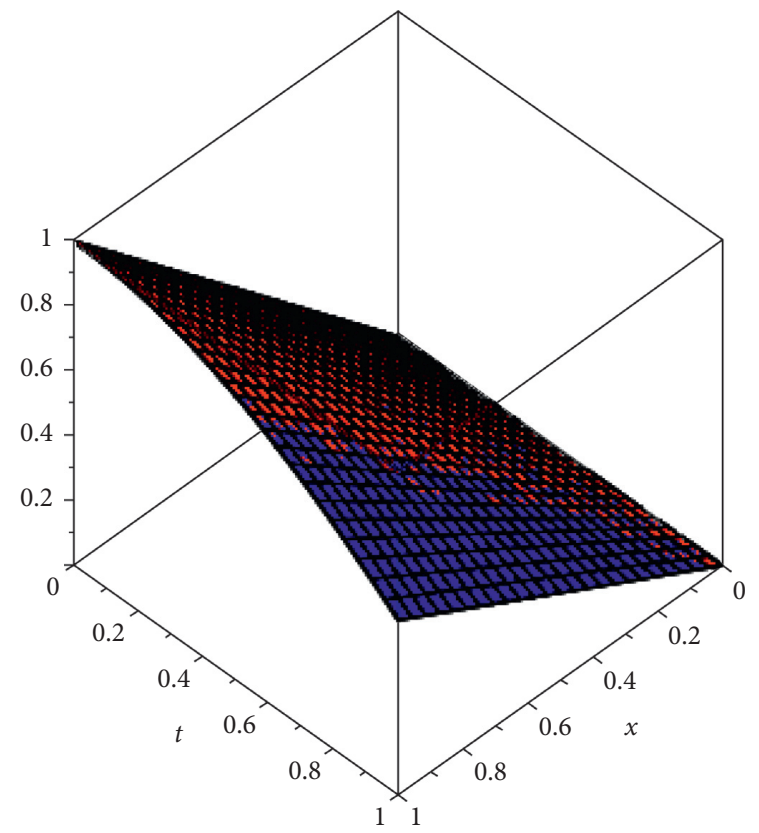

Figure 4: Comparison of the exact solution (blue) and the approximate solutions (red) of Example 4. 


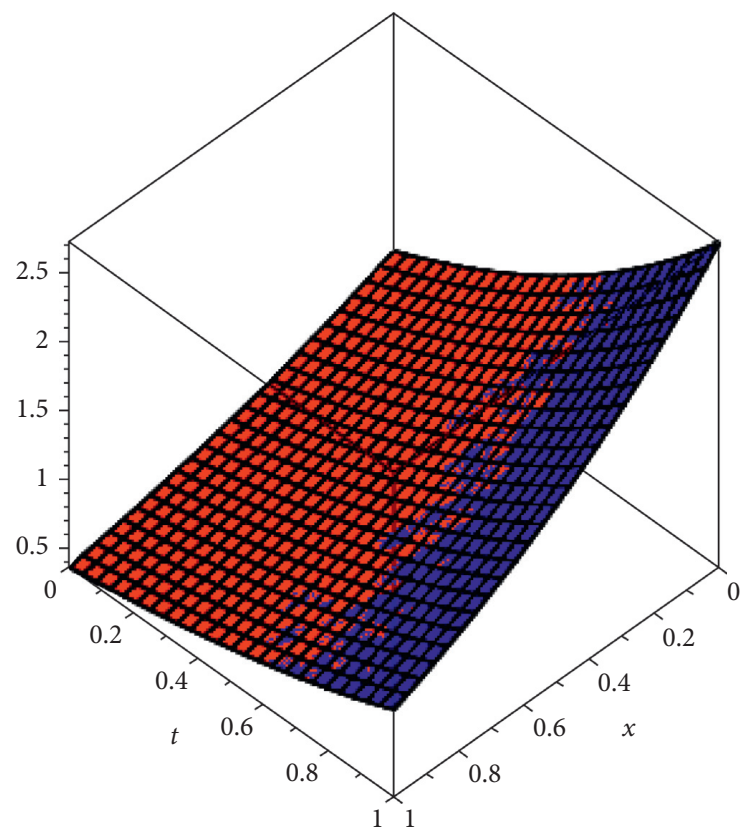

Figure 5: Comparison of the exact solution (blue) and the approximate solutions (red) of Example 5 for $\alpha=1$.

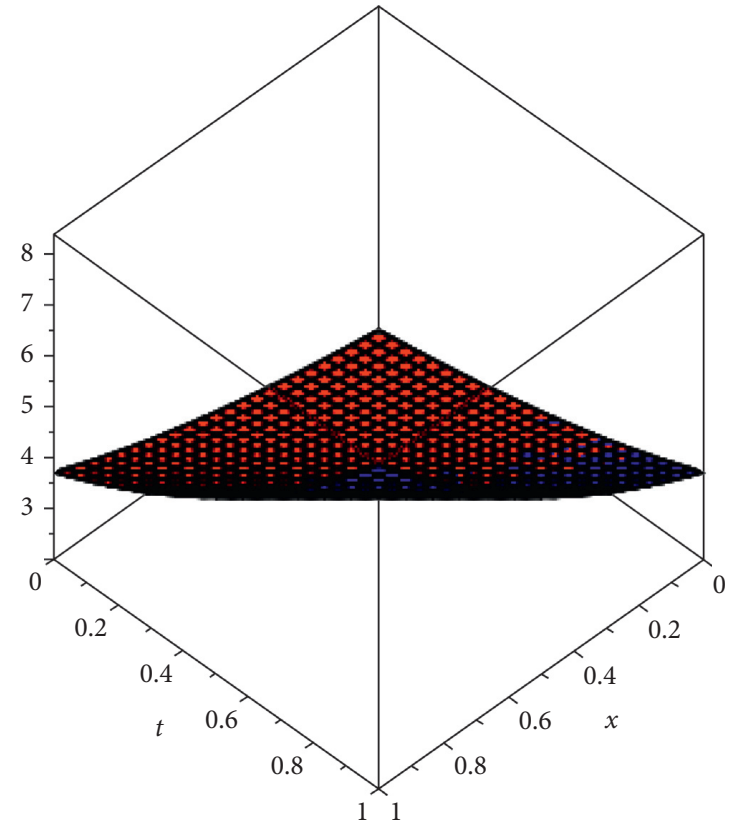

(a)

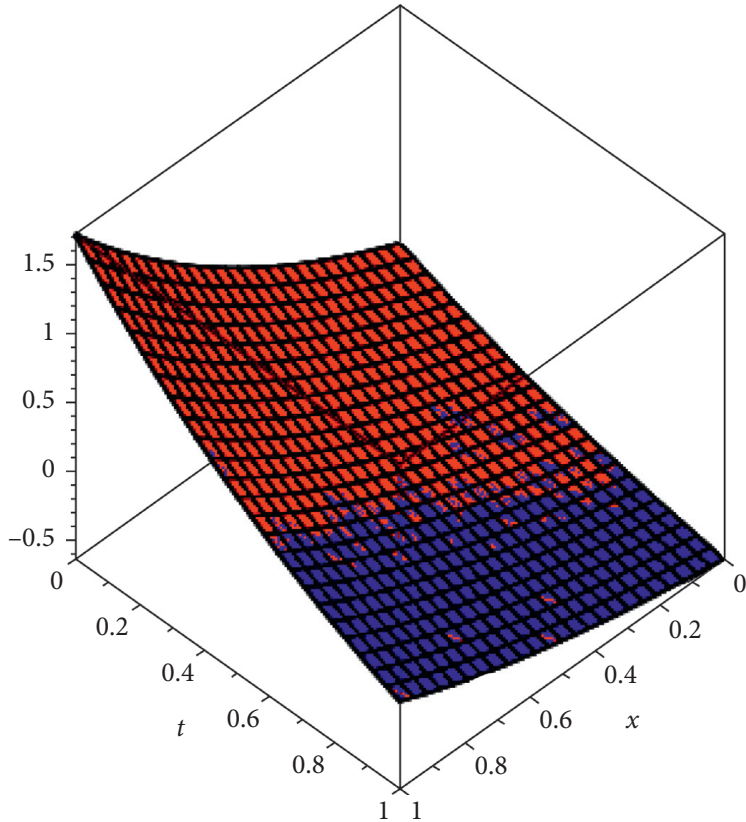

(b)

Figure 6: Comparison of the exact solution (blue) and the approximate solutions (red) of Example 6. (a) The solution of $w_{n}(x, t)$. (b) The solution of $v_{n}(x, t)$. 


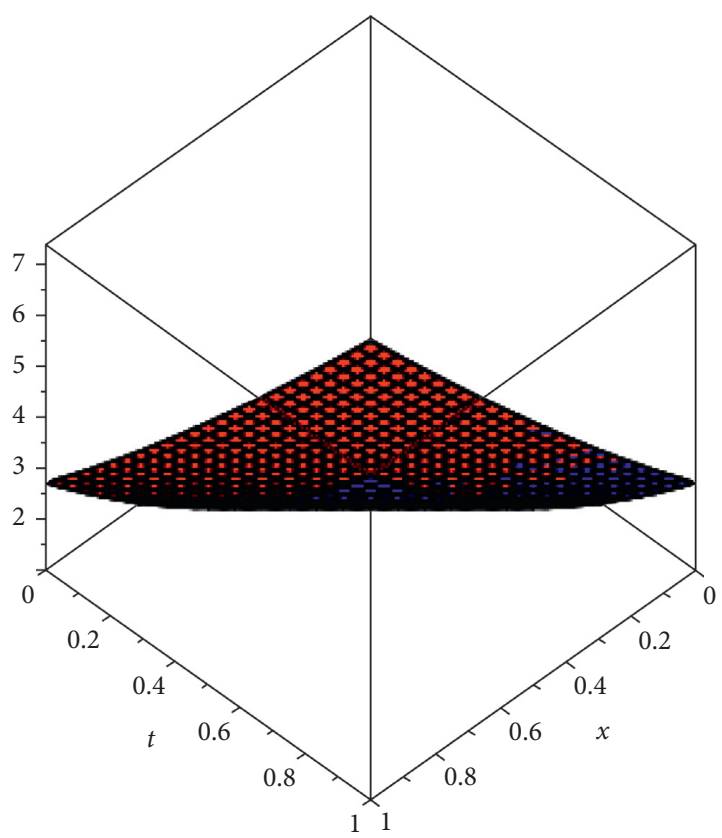

(a)

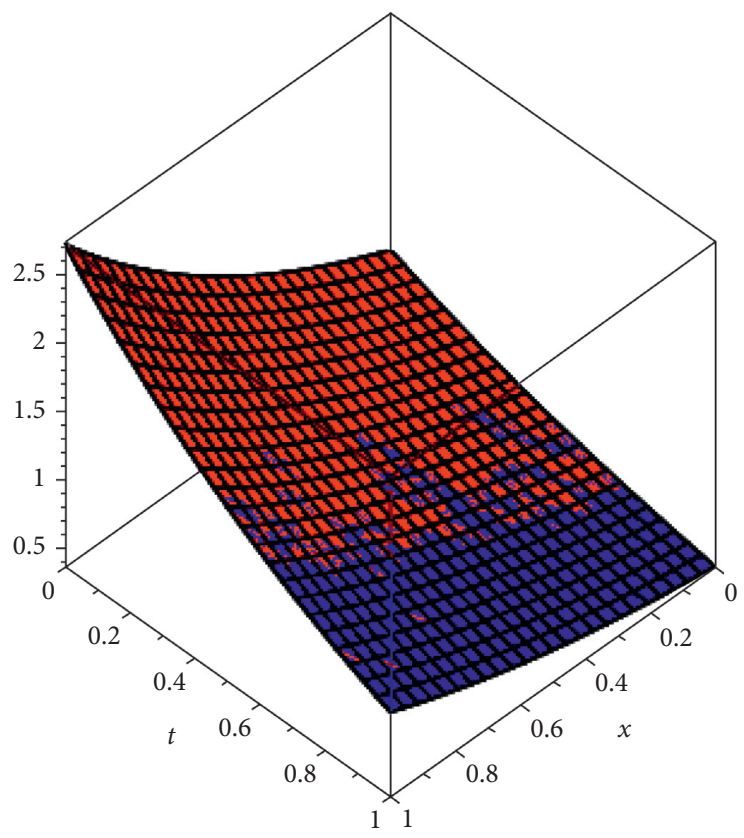

(b)

Figure 7: Comparison of the exact solution (blue) and the approximate solutions (red) of Example 7. (a) The solution of $w_{n}(x, t)$. (b) The solution of $v_{n}(x, t)$.

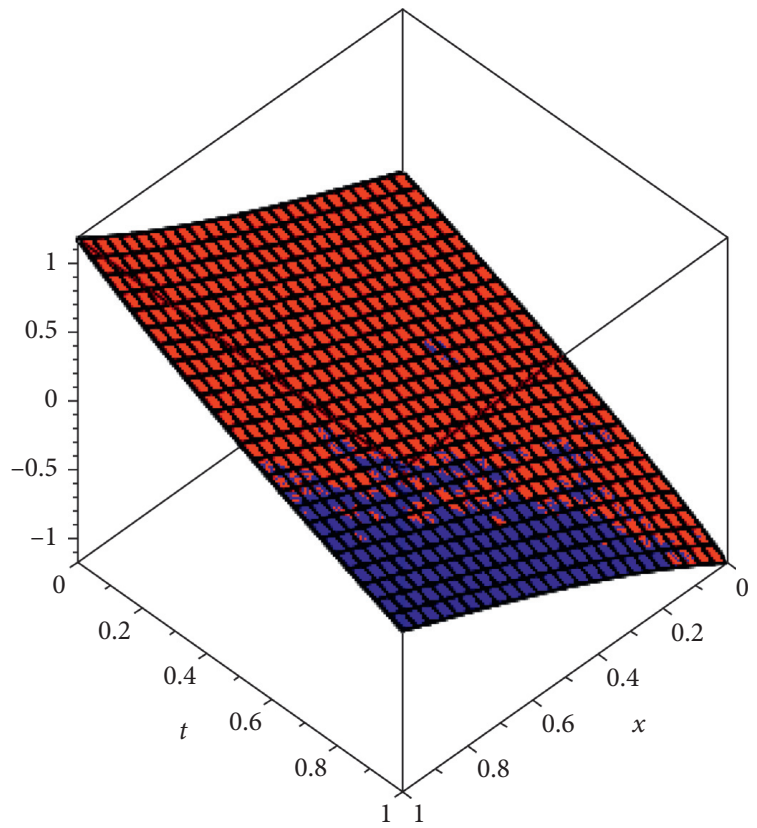

(a)

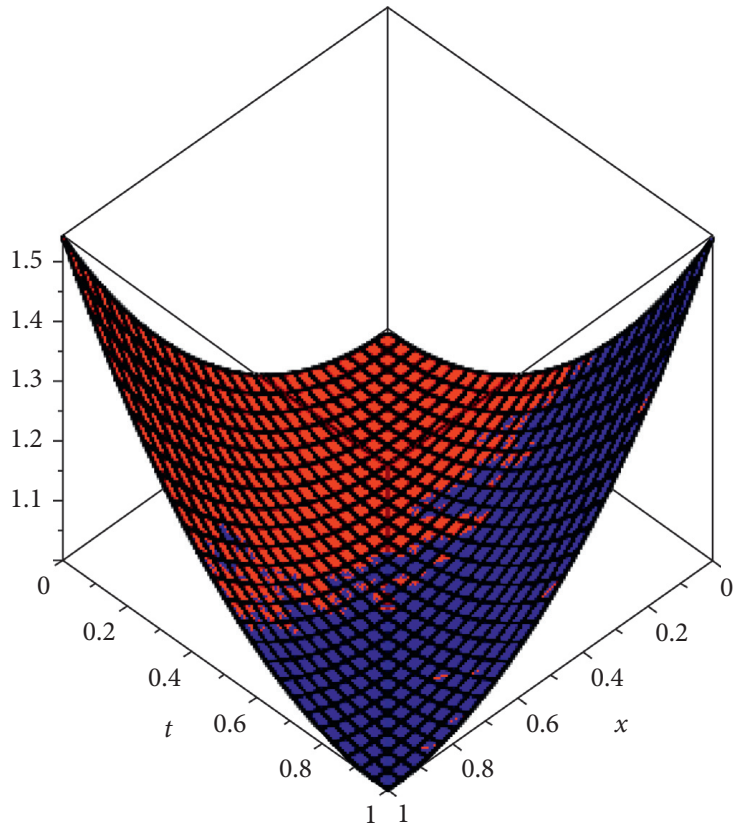

(b)

Figure 8: Comparison of the exact solution (blue) and the approximate solutions (red) of Example 8 for $\alpha=1$. (a) The solution of $w_{n}(x, t)$. (b) The solution of $v_{n}(x, t)$. 
TABLe 2: Numerical result of Example 1.

\begin{tabular}{|c|c|c|c|c|c|}
\hline$t$ & $u_{E}$ & $u_{7, R}$ & $u_{9, R}$ & $n=7, e(u)$ & $n=9, e(u)$ \\
\hline 0 & 0 & 0 & 0 & 0 & 0 \\
\hline 0.2 & 0.1986693308 & 0.1986693309 & 0.1986693309 & $7.02 \times 10^{-12}$ & $5.6 \times 10^{-12}$ \\
\hline 0.4 & 0.3894183423 & 0.3894183415 & 0.3894183422 & $7.12 \times 10^{-10}$ & $6.36 \times 10^{-12}$ \\
\hline 0.6 & 0.5646424734 & 0.5646424457 & 0.5646424735 & $2.77 \times 10^{-8}$ & $7.57 \times 10^{-11}$ \\
\hline 0.8 & 0.7173560909 & 0.7173557232 & 0.7173560931 & $3.68 \times 10^{-7}$ & $2.13 \times 10^{-9}$ \\
\hline 1 & 0.8414709848 & 0.8414682539 & 0.8414710096 & $2.73 \times 10^{-6}$ & $2.49 \times 10^{-8}$ \\
\hline
\end{tabular}

TABle 3: Numerical result of Example 2.

\begin{tabular}{|c|c|c|c|c|c|}
\hline$t$ & $u_{E}$ & $u_{7, R}$ & $u_{9, R}$ & $n=7, e(u)$ & $n=9, e(u)$ \\
\hline 0 & 0 & 0 & 0 & 0 & 0 \\
\hline 0.2 & 0.2027100355 & 0.2027100242 & 0.2027100354 & $1.14 \times 10^{-8}$ & $1.77 \times 10^{-10}$ \\
\hline 0.4 & 0.4227932187 & 0.4227870882 & 0.4227928212 & $6.13 \times 10^{-6}$ & $3.97 \times 10^{-7}$ \\
\hline 0.6 & 0.6841368083 & 0.6838787657 & 0.6840991598 & $2.58 \times 10^{-4}$ & $3.76 \times 10^{-5}$ \\
\hline 0.8 & 1.029638557 & 1.025675296 & 1.028610569 & $3.96 \times 10^{-3}$ & $1.03 \times 10^{-3}$ \\
\hline 1 & 1.557407725 & 1.520634920 & 1.542504409 & $3.68 \times 10^{-2}$ & $1.49 \times 10^{-2}$ \\
\hline
\end{tabular}

TABle 4: Numerical result of Example 3.

\begin{tabular}{|c|c|c|c|c|c|}
\hline$(x, t)$ & $u_{E}$ & $u_{7, R}$ & $u_{9, R}$ & $n=7, e(u)$ & $n=9, e(u)$ \\
\hline$(0,0)$ & 0 & 0 & 0 & 0 & 0 \\
\hline$(0.2,0.1)$ & 0.3333333334 & 0.33333248 & 0.3333332992 & $8.53 \times 10^{-7}$ & $3.41 \times 10^{-8}$ \\
\hline$(0.4,0.2)$ & 0.5714285714 & 0.57105408 & 0.5713686528 & $3.74 \times 10^{-4}$ & $5.9 \times 10^{-5}$ \\
\hline$(0.6,0.3)$ & 0.75 & 0.73740288 & 0.7454650368 & $1.26 \times 10^{-2}$ & $4.53 \times 10^{-3}$ \\
\hline$(0.8,0.4)$ & 0.8888888889 & 0.73975808 & 0.7934451712 & $1.49 \times 10^{-1}$ & $9.54 \times 10^{-2}$ \\
\hline
\end{tabular}

TABle 5: Numerical result of Example 4.

\begin{tabular}{|c|c|c|c|c|c|}
\hline$(x, t)$ & $u_{E}$ & $u_{6, R}$ & $u_{10, R}$ & $n=6, e(u)$ & $n=10, e(u)$ \\
\hline$(0.1,0)$ & 0.1 & 0.1 & 0.1 & 0 & 0 \\
\hline$(0.3,0.2)$ & 0.2940199733 & 0.2940199733 & 0.2940199734 & $3.33 \times 10^{-11}$ & $5.24 \times 10^{-11}$ \\
\hline$(0.5,0.4)$ & 0.4605304970 & 0.4605304889 & 0.4605304970 & $8.11 \times 10^{-9}$ & $1.29 \times 10^{-12}$ \\
\hline$(0.7,0.6)$ & 0.5777349304 & 0.5777346400 & 0.5777349304 & $2.9 \times 10^{-7}$ & $3.36 \times 10^{-11}$ \\
\hline$(0.9,0.8)$ & 0.6270360384 & 0.6270323200 & 0.6270360383 & $3.72 \times 10^{-6}$ & $1.16 \times 10^{-10}$ \\
\hline
\end{tabular}

TAвLE 6: Numerical result of Example 5.

\begin{tabular}{|c|c|c|c|c|c|}
\hline$(x, t)$ & $u_{E}$ & $u_{7, R}$ & $u_{10, R}$ & $n=7, e(u)$ & $n=10, e(u)$ \\
\hline$(0.1,0)$ & 0.9048374180 & 0.9048374180 & 0.9048374180 & 0 & 0 \\
\hline$(0.3,0.2)$ & 0.9048374180 & 0.9048374187 & 0.9048374187 & $2.34 \times 10^{-11}$ & $2.47 \times 10^{-11}$ \\
\hline$(0.5,0.4)$ & 0.9048374180 & 0.9048374079 & 0.9048374182 & $1.03 \times 10^{-8}$ & $3.13 \times 10^{-11}$ \\
\hline$(0.7,0.6)$ & 0.9048374180 & 0.9048371964 & 0.9048374184 & $2.21 \times 10^{-7}$ & $2.38 \times 10^{-11}$ \\
\hline$(0.9,0.8)$ & 0.9048374180 & 0.9048355630 & 0.9048374173 & $1.85 \times 10^{-6}$ & $9.49 \times 10^{-10}$ \\
\hline
\end{tabular}

$$
\left\{\begin{array}{l}
\frac{\partial^{\alpha} w}{\partial t^{\alpha}}-\frac{\partial v}{\partial x}+v+w=0 \\
\frac{\partial^{\beta} v}{\partial t^{\beta}}-\frac{\partial w}{\partial x}+v+w=0
\end{array}\right.
$$

which subject to the initial conditions:

$$
\left\{\begin{array}{l}
w(x, 0)=\sinh x \\
v(x, 0)=\cosh x
\end{array}\right.
$$


TABLe 7: Numerical result of Example 6.

\begin{tabular}{|c|c|c|c|c|c|}
\hline$(x, t)$ & $u_{E}$ & $u_{7, R}$ & $u_{10, R}$ & $n=7, e(u)$ & $n=10, e(u)$ \\
\hline$(0,0.1)$ & 2.105170918 & 2.105170918 & 2.105170918 & $7.54 \times 10^{-11}$ & $7.57 \times 10^{-11}$ \\
\hline$(0.2,0.3)$ & 2.648721271 & 2.648721267 & 2.648721270 & $2.57 \times 10^{-9}$ & $5.14 \times 10^{-10}$ \\
\hline$(0.4,0.5)$ & 3.459603111 & 3.459602959 & 3.459603111 & $1.52 \times 10^{-7}$ & $6.81 \times 10^{-10}$ \\
\hline$(0.6,0.7)$ & 4.669296668 & 4.669293844 & 4.669296666 & $2.82 \times 10^{-6}$ & $2.09 \times 10^{-9}$ \\
\hline$(0.8,0.9)$ & 6.473947392 & 6.473921020 & 6.473947370 & $2.64 \times 10^{-5}$ & $2.06 \times 10^{-8}$ \\
\hline$(x, t)$ & $v_{E}$ & $v_{7, R}$ & $v_{10, R}$ & $n=7, e(v)$ & $n=10, e(v)$ \\
\hline$(0,0.1)$ & $-0.951625820 e-1$ & $-0.951625819 e-1$ & $-0.951625819 e-1$ & $3.57 \times 10^{-11}$ & $3.59 \times 10^{-11}$ \\
\hline$(0.2,0.3)$ & $-0.951625820 e-1$ & $-0.951625840 e-1$ & $-0.951625822 e-1$ & $2.01 \times 10^{-9}$ & $8.46 \times 10^{-11}$ \\
\hline$(0.4,0.5)$ & $-0.951625820 e-1$ & $-0.951627186 e-1$ & $-0.951625816 e-1$ & $1.37 \times 10^{-7}$ & $2.19 \times 10^{-10}$ \\
\hline$(0.6,0.7)$ & $-0.951625820 e-1$ & $-0.951649979 e-1$ & $-0.951625810 e-1$ & $2.42 \times 10^{-6}$ & $6.6 \times 10^{-10}$ \\
\hline$(0.8,0.9)$ & $-0.951625820 e-1$ & $-0.951841642 e-1$ & $-0.951625658 e-1$ & $2.16 \times 10^{-5}$ & $1.62 \times 10^{-8}$ \\
\hline
\end{tabular}

TABLe 8: Numerical result of Example 7.

\begin{tabular}{lccccc}
\hline$(x, t)$ & $u_{E}$ & $u_{7, R}$ & $u_{10, R}$ & $n=7, e(u)$ & $n=10, e(u)$ \\
\hline$(0,0.3)$ & 1.349858808 & 1.349858805 & 1.349858807 & $2.11 \times 10^{-9}$ & $4.23 \times 10^{-10}$ \\
$(0.2,0.4)$ & 1.822118800 & 1.822118780 & 1.822118801 & $2.06 \times 10^{-8}$ & $1.55 \times 10^{-10}$ \\
$(0.4,0.5)$ & 2.459603111 & 2.459602959 & 2.459603111 & $1.52 \times 10^{-7}$ & $6.81 \times 10^{-10}$ \\
$(0.6,0.6)$ & 3.320116923 & 3.320116109 & 3.320116923 & $8.14 \times 10^{-7}$ & $1.14 \times 10^{-9}$ \\
$(0.8,0.7)$ & 4.481689070 & 4.481685621 & 4.481689068 & $3.45 \times 10^{-6}$ & $1.37 \times 10^{-9}$ \\
\hline$(x, t)$ & $v_{E}$ & $v_{7, R}$ & $v_{10, R}$ & $n=7, e(v)$ & $n=10, e(v)$ \\
\hline$(0,0.3)$ & 0.7408182207 & 0.7408182191 & 0.7408182206 & $1.59 \times 10^{-9}$ & $1.92 \times 10^{-11}$ \\
$(0.2,0.4)$ & 0.8187307531 & 0.8187307340 & 0.8187307530 & $1.91 \times 10^{-8}$ & $1.32 \times 10^{-10}$ \\
$(0.4,0.5)$ & 0.9048374180 & 0.9048372814 & 0.9048374184 & $1.37 \times 10^{-7}$ & $2.19 \times 10^{-10}$ \\
$(0.6,0.6)$ & 1 & 0.9999992884 & 1 & $7.11 \times 10^{-7}$ & $6.62 \times 10^{-11}$ \\
$(0.8,0.7)$ & 1.105170918 & 1.105167967 & 1.105170919 & $2.95 \times 10^{-6}$ & $4.99 \times 10^{-10}$ \\
\hline
\end{tabular}

TABLe 9: Numerical result of Example 8.

\begin{tabular}{|c|c|c|c|c|c|}
\hline$(x, t)$ & $u_{E}$ & $u_{7, R}$ & $u_{9, R}$ & $n=7, e(u)$ & $n=9, e(u)$ \\
\hline$(0.1,0.1)$ & 0 & 0 & 0 & $3.5 \times 10^{-11}$ & $3.5 \times 10^{-11}$ \\
\hline$(0.2,0.3)$ & -0.1001667500 & -0.1001667505 & -0.1001667502 & $4.17 \times 10^{-10}$ & $1.73 \times 10^{-10}$ \\
\hline$(0.3,0.5)$ & -0.2013360025 & -0.2013360265 & -0.2013360026 & $2.4 \times 10^{-8}$ & $8.6 \times 10^{-11}$ \\
\hline$(0.4,0.7)$ & -0.3045202934 & -0.3045207633 & -0.3045202961 & $4.7 \times 10^{-7}$ & $2.88 \times 10^{-9}$ \\
\hline$(0.5,0.9)$ & -0.4107523258 & -0.4107567262 & -0.4107523679 & $4.4 \times 10^{-6}$ & $4.1 \times 10^{-8}$ \\
\hline$(x, t)$ & $v_{E}$ & $v_{7, R}$ & $v_{9, R}$ & $n=7, e(v)$ & $n=9, e(v)$ \\
\hline$(0.1,0.1)$ & 1 & 1 & 1 & $4.76 \times 10^{-11}$ & $4.76 \times 10^{-11}$ \\
\hline$(0.2,0.3)$ & 1.005004168 & 1.005004166 & 1.005004168 & $1.18 \times 10^{-9}$ & $4.67 \times 10^{-10}$ \\
\hline$(0.3,0.5)$ & 1.020066756 & 1.020066655 & 1.020066756 & $1.01 \times 10^{-7}$ & $8.15 \times 10^{-10}$ \\
\hline$(0.4,0.7)$ & 1.045338514 & 1.045337006 & 1.045338506 & $1.51 \times 10^{-6}$ & $7.99 \times 10^{-9}$ \\
\hline$(0.5,0.9)$ & 1.081072372 & 1.081060784 & 1.081072265 & $1.16 \times 10^{-5}$ & $1.05 \times 10^{-7}$ \\
\hline
\end{tabular}


According to the operations of differential transformation given in Table 1 for equation (82), we obtain the following recurrent relation:

$$
\begin{aligned}
& \frac{\Gamma(\alpha(k+1)+1)}{\Gamma(\alpha k+1)} \mathscr{W}_{k+1}^{\alpha}(x)-\frac{\partial}{\partial x} \mathscr{V}_{k}(x)+\mathscr{V}_{k}(x)+\mathscr{W}_{k}(x)=0, \\
& \frac{\Gamma(\beta(k+1)+1)}{\Gamma(\beta k+1)} \mathscr{V}_{k+1}^{\beta}(x)-\frac{\partial}{\partial x} \mathscr{W}_{k}(x)+\mathscr{V}_{k}(x)+\mathscr{W}_{k}(x)=0,
\end{aligned}
$$

and from initial conditions (83), we write

$$
\begin{aligned}
& \mathscr{W}_{0}^{\alpha}(x)=\sinh x, \\
& \mathscr{V}_{0}^{\beta}(x)=\cosh x .
\end{aligned}
$$

Substituting the above equations in equation (84), we drive the following results:

$$
\begin{aligned}
\mathscr{W}_{1}^{\alpha}(x)= & -\frac{\cosh x}{\Gamma(\alpha+1)}, \mathscr{W}_{2}^{\alpha}(x)=\frac{\sinh x}{\Gamma(2 \alpha+1)}, \mathscr{W}_{3}^{\alpha}(x) \\
& =-\frac{\cosh x}{\Gamma(3 \alpha+1)}, \ldots, \\
\mathscr{V}_{1}^{\beta}(x)= & -\frac{\sinh x}{\Gamma(\beta+1)}, \mathscr{V}_{2}^{\beta}(x)=\frac{\cosh x}{\Gamma(2 \beta+1)}, \mathscr{V}_{3}^{\beta}(x) \\
& =-\frac{\sinh x}{\Gamma(3 \beta+1)}, \ldots
\end{aligned}
$$

Hence, the solutions in the series form are as follows:

$$
\begin{aligned}
\widetilde{w}_{n}(x, t) & =\sum_{k=0}^{\infty} \mathscr{W}_{k}^{\alpha}(x) t^{\alpha k}=\mathscr{W}_{0}^{\alpha}(x)+\mathscr{W}_{1}^{\alpha}(x) t^{\alpha}+\mathscr{W}_{2}^{\alpha}(x) t^{2 \alpha}+\mathscr{W}_{3}^{\alpha}(x) t^{3 \alpha}+\cdots \\
& =\sinh x+\frac{(-1) t^{\alpha}}{\Gamma(\alpha+1)} \cosh x+\frac{(-1)^{2} t^{2 \alpha}}{\Gamma(2 \alpha+1)} \sinh x+\frac{(-1)^{3} t^{3 \alpha}}{\Gamma(3 \alpha+1)} \cosh x+\cdots, \\
\widetilde{v}_{n}(x, t) & =\sum_{k=0}^{\infty} \mathscr{V}_{k}^{\beta}(x) t^{\beta k}=\mathscr{V}_{0}^{\beta}(x)+\mathscr{V}_{1}^{\beta}(x) t^{\beta}+\mathscr{V}_{2}^{\beta}(x) t^{2 \beta}+\mathscr{V}_{3}^{\beta}(x) t^{3 \beta}+\cdots \\
& =\cosh x+\frac{(-1) t^{\beta}}{\Gamma(\beta+1)} \sinh x+\frac{(-1)^{2} t^{2 \beta}}{\Gamma(2 \beta+1)} \cosh x+\frac{(-1)^{3} t^{3 \beta}}{\Gamma(3 \beta+1)} \sinh x+\cdots
\end{aligned}
$$

which for $\alpha=\beta=1$ converges efficiently to the exact solutions $w(x, t)=\sinh (x-t)$ and $v(x, t)=\cosh (x-t)$.

Results for Examples 1-8 are reported in Figures 1-8 and Tables 2-9, respectively. In these tables, the terms $w_{E}, v_{E}$, $w_{n, R}, v_{n, R}$, and $e(w), e(v)$ stand for exact solution, $n$th order approximate solution of RDTM, and their absolute error, respectively.

\section{Conclusions}

In this work, the convergence of the reduced differential transform method (RDTM) for solving the linear and nonlinear ordinary, partial, fractional differential equations, and their systems is discussed. The main strength of the RDTM is its fast convergence, and under the assumption of Theorem 1, the method is convergent to the exact solution of the problem. The sufficient condition for convergence of the method and an error estimate has been addressed. For the efficiency of the RDTM, the form of the initial approximation is very important. We note that the RDTM solutions computed via a simple algorithm and without involving the perturbation, linearization, or discretization provide a solution in both numerical and analytical manner. RDTM can be applied in most of the biological, physical, engineering, etc. models as an alternative for obtaining reliable and fastest convergence and useful approximations. Thus, it can be concluded that the RDTM is a simple and powerful tool for solving functional equations.

\section{Data Availability}

No data were used to support this study.

\section{Conflicts of Interest}

The authors declare that there are no conflicts of interest regarding the publication of this paper.

\section{References}

[1] Y. Keskin, Ph.D. thesis, Selcuk University, Konya, Turkish, 2010.

[2] Y. Keskin and G. Oturanc, "Reduced differential transform method for partial differential equations," International Journal of Nonlinear Sciences and Numerical Simulation, vol. 10, no. 6, pp. 741-749, 2009.

[3] Y. Keskin and G. Oturanc, "The reduced differential transform method: a new approach to fractional partial differential equations," Nonlinear Science Letters A, vol. 1, no. 2, pp. 207-217, 2010.

[4] Y. Keskin and G. Oturanc, "The reduced differential transformation method for solving linear and nonlinear wave 
equations," Iranian Journal of Science and Technology, vol. 34, no. 2, pp. 113-122, 2010.

[5] M. A. Abdou, "Fractional reduced differential transform method and its applications," Journal of Nonlinear Sciences and Numerical Simulation, vol. 26, pp. 55-64, 2018.

[6] M. O. Al-Amr, "New applications of reduced differential transform method," Alexandria Engineering Journal, vol. 53, no. 1, pp. 243-247, 2014.

[7] M. S. Mohamed and K. A. Gepreel, "Reduced differential transform method for nonlinear integral member of Kadomtsev-Petviashvili hierarchy differential equations," Journal of the Egyptian Mathematical Society, vol. 25, no. 1, pp. 1-7, 2017.

[8] R. K. Saeed and A. A. Mustafa, Numerical Solution of FisherKPP Equation by Using Reduced Differential Transform Method, AIP Publishing, Melville, NY, USA, 2017.

[9] V. K. Srivastava, M. K. Awasthi, and S. Kumar, "Analytical approximations of two and three dimensional time-fractional telegraphic equation by reduced differential transform method," Egyptian Journal of Basic and Applied Sciences, vol. 1, no. 1, pp. 60-66, 2014.

[10] R. Abazari, "Numerical simulation of coupled nonlinear Schrödinger equation by RDTM and comparison with DTM," Journal of Applied Sciences, vol. 11, no. 20, pp. 3454-3463, 2011.

[11] O. Acan, O. Firat, and Y. Keskin, "Conformable variational iteration method, conformable fractional reduced differential transform method and conformable homotopy analysis method for nonlinear fractional partial differential equations," Waves Random Complex Media, vol. 30, no. 2, pp. 250-268, 2018.

[12] Z. Cui, Z. Mao, S. Yang, and P. Yu, "Approximate analytical solutions of fractional perturbed diffusion equation by reduced differential transform method and the homotopy perturbation method," Mathematical Problems in Engineering, vol. 2013, Article ID 186934, , 2013.

[13] P. K. Gupta, “Approximate analytical solutions of fractional Benney-Lin equation by reduced differential transform method and the homotopy perturbation method," Computers \& Mathematics with Applications, vol. 61, no. 9, pp. 28292842, 2011.

[14] A. Saravanan and N. Magesh, "A comparison between the reduced differential transform method and the Adomian decomposition method for the Newell-Whitehead-Segel equation," Journal of the Egyptian Mathematical Society, vol. 21, no. 3, pp. 259-265, 2013.

[15] J. Biazar and M. Eslami, "Differential transform method for quadratic riccati differential equation," International Journal of Nonlinear Science, vol. 9, no. 4, pp. 444-447, 2010.

[16] B. K. Singh and Mahendra, "A numerical computation of a system of linear and nonlinear time dependent partial differential equations using reduced differential transform method," International Journal of Differential Equations, vol. 2016, Article ID 4275389, 8 pages, 2016.

[17] N. Taghizadeh and S. R. Moosavi Noori, "Exact solutions of the cubic nonlinear Schrodinger equation with a trapping potential by reduced differential transform method," Mathematical Sciences Letters, vol. 5, no. 3, pp. 1-5, 2016.

[18] N. Taghizadeh and S. R. M. Noori, "Reduced differential transform method for solving parabolic-like and hyperboliclike equations," SeMA Journal, vol. 74, no. 4, pp. 559-567, 2017.

[19] O. Acan, M. Mohamed Al Qurashi, and D. Baleanu, "Reduced differential transform method for solving time and space local fractional partial differential equations," Journal of Nonlinear Sciences and Applications, vol. 10, no. 10, pp. 5230-5238, 2017.

[20] M. Garg, P. Manohar, and S. L. Kalla, "Generalized differential transform method to space-time fractional telegraph equation," International Journal of Differential Equations, vol. 2011, Article ID 548982, 9 pages, 2011.

[21] J. G. Liu, X. J. Yang, Y. Y. Feng, and P. Cui, "On the $(N+1)$ dimensional local fractional reduced differential transform method and its applications," Mathematical Methods in the Applied Sciences, vol. 43, no. 15, pp. 8856-8866, 2020.

[22] S. Saha Ray, "Numerical solutions and solitary wave solutions of fractional $\mathrm{KdV}$ equations using modified fractional reduced differential transform method," Computational Mathematics and Mathematical Physics, vol. 53, no. 12, pp. 1870-1881, 2013.

[23] M. Valizadeh, Y. Mahmoudi, and F. Dastmalchi Saei, “Application of natural transform method to fractional pantograph delay differential equations," Journal of Mathematics, vol. 2019, Article ID 3913840, 9 pages, 2019.

[24] M. Bakhshi and M. Asghari-Larimi, "Three dimensional differential transform method for solving nonlinear threedimensional Volterra integral equations," Journal of Mathematics and Computer Science, vol. 4, no. 2, pp. 246-256, 2012.

[25] S. R. Moosavi Noori and N. Taghizadeh, "Application of reduced differential transform method for solving two-dimensional Volterra integral equations of the second kind," Applications and Applied Mathematics, vol. 14, pp. 1003-1019, 2019.

[26] A. Ziqan, S. Armiti, and I. Suwan, "Solving three-dimensional Volterra integral equation by the reduced differential transform method," International Journal of Applied Mathematical Research, vol. 5, no. 2, pp. 103-106, 2016.

[27] A. Arikoglu and I. Ozkol, "Solutions of integral and integrodifferential equation systems by using differential transform method," Computers \& Mathematics with Applications, vol. 56, no. 9, pp. 2411-2417, 2008.

[28] S. R. Moosavi Noori and N. Taghizadeh, "Study on solving two-dimensional linear and nonlinear Volterra partial integro-differential equations by reduced differential transform method," Applications and Applied Mathematics, vol. 15, no. 1, pp. 394-407, 2020.

[29] A. Tari and S. Shahmorad, "Differential transform method for the system of two-dimensional nonlinear Volterra integrodifferential equations," Computers \& Mathematics with Applications, vol. 61, no. 9, pp. 2621-2629, 2011.

[30] M. Caputo, "Linear models of dissipation whose Q is almost frequency independent-II," Geophysical Journal International, vol. 13, no. 5, pp. 529-539, 1967.

[31] M. Caputo, Elasticita e Dissipazione. Zanichelli, Bologna, Italy, 1969.

[32] M. Caputo and F. Mainardi, "Linear models of dissipation in anelastic solids," La Rivista del Nuovo Cimento, vol. 1, no. 2, pp. 161-198, 1971.

[33] H. F. Ahmed, M. S. M. Bahgat, and M. Zaki, "Numerical approaches to system of fractional partial differential equations," Journal of the Egyptian Mathematical Society, vol. 25, no. 2, pp. 141-150, 2017.

[34] I. Podlubny, Fractional Dierential Equations. Vol. 198 of Mathematics in Science and Engineering, Academic Press, New York, NY, USA, 1999.

[35] D. Zeidan, C. K. Chau, T. T. Lu, and W. Q. Zheng, "Mathematical studies of the solution of Burgers' equations by Adomian decomposition method," Mathematical Methods in the Applied Sciences, pp. 1-18, 2019. 
[36] M. S. H. Chowdhury and I. Hashim, "Application of homotopy-perturbation method to Klein-Gordon and sineGordon equations," Chaos, Solitons \& Fractals, vol. 39, pp. 1928-1935, 2009.

[37] A. Akgül, A. Cordero, and J. R. Torregrosa, "Solutions of fractional gas dynamics equation by a new technique," Mathematical Methods in the Applied Sciences, vol. 43, no. 3, pp. 1349-1358, 2019.

[38] H. Thabet and S. Kendre, "Analytical solutions for conformable space-time fractional partial differential equations via fractional differential transform," Chaos, Solitons \& Fractals, vol. 109, pp. 238-245, 2018.

[39] H. Jafari, M. Nazari, D. Baleanu, and C. M. Khalique, "A new approach for solving a system of fractional partial differential equations," Computers \& Mathematics with Applications, vol. 66, no. 5, pp. 838-843, 2013.

[40] H. Jafari and S. Seifi, "Solving a system of nonlinear fractional partial differential equations using homotopy analysis method," Communications in Nonlinear Science and Numerical Simulation, vol. 14, no. 5, pp. 1962-1969, 2009. 